\title{
ON THE NORMAL STRUCTURE COEFFICIENT AND THE BOUNDED SEQUENCE COEFFICIENT
}

\author{
TECK-CHEONG LIM
}

\begin{abstract}
The two notions of normal structure coefficient and bounded sequence coefficient introduced by Bynum are shown to be the same. A lower bound for the normal structure coefficient in $L^{p}, p>2$, is also given.
\end{abstract}

Let $X$ be a Banach space and $C$ a closed convex bounded subset of $X$. For each $x$ in $C$, let $R(x, C)=\sup \{\|x-y\|: y$ in $C\}$ and let $R(C)$ denote the Chebyshev radius of $C[2, \mathrm{p} .178]$ :

$$
R(C)=\inf \{R(x, C): x \text { in } C\} .
$$

Let $D(C)$ denote the diameter of $C, D(C)=\sup \{\|x-y\|: x, y \in C\}$.

For a bounded sequence $\left\{x_{n}\right\}$ in $X$, the asymptotic diameter $A\left(\left\{x_{n}\right\}\right)$ of $\left\{x_{n}\right\}$ is defined to be $\lim _{n} \sup \left\{\left\|x_{k}-x_{m}\right\|: m \geqslant n, k \geqslant n\right\}$.

In [1], Bynum introduced the following two coefficients of $X$, called the normal structure coefficient and the bounded sequence coefficient respectively:

$$
N(X)=\inf \{D(C) / R(C): C \text { closed convex bounded nonempty }
$$

$$
\text { subsets of } X \text { with }|C|>1\} \text {, }
$$

$B S(X)=\sup \left\{M:\right.$ for every bounded sequence $\left\{x_{n}\right\}$ in $X$, there

$$
\text { exists } \left.y \text { in } \overline{\operatorname{Co}}\left(x_{n}\right) \text { such that } M \lim _{n} \sup \left\|x_{n}-y\right\| \leqslant A\left(\left\{x_{n}\right\}\right)\right\} \text {. }
$$

Another coefficient relating to the asymptotic radius of a sequence (see e.g. [3]) can be defined as follows: Let $\left\{x_{n}\right\}$ be a bounded sequence in $X$. For each $x$ in $X$, define

$$
r\left(x,\left\{x_{n}\right\}\right)=\underset{n}{\lim \sup _{n}\left\|x_{n}-x\right\| .}
$$

The number $r\left(\left\{x_{n}\right\}\right)=\inf \left\{f\left(x,\left\{x_{n}\right\}\right): x \in \overline{\mathrm{Co}}\left(x_{n}\right)\right\}$ will be called the asymptotic radius of $\left\{x_{n}\right\}$, or more precisely, the asymptotic radius of $\left\{x_{n}\right\}$ w.r.t. $\overline{\operatorname{Co}}\left(x_{n}\right)$. We shall denote the coefficient

$$
\inf \left\{A\left(\left\{x_{n}\right\}\right) / r\left(\left\{x_{n}\right\}\right):\left\{x_{n}\right\} \text { bounded nonconvergent sequences in } X\right\}
$$

by $A(X)$.

Received by the editors April 13, 1982 and, in revised form, July 23, 1982.

1980 Mathematics Subject Classification. Primary 46B20, 47H09, 47H10; Secondary 52A05.

Key words and phrases. Normal structure coefficient, bounded sequence coefficient, Chebyshev radius, asymptotic radius, inequality in $L^{p}$. 
In [1], Bynum mentioned that the two coefficients $N(X)$ and $B S(X)$ are equal in a separable Banach space $X$. In this note, we shall show that the three coefficients are equal in any Banach space $X$.

TheOREM 1. For a Banach space $X, N(X)=B S(X)=A(X)$.

Proof. It follows readily from the definition that $B S(X)=A(X)$. Indeed, we may assume that the sequences in the definition of $B S(X)$ are nonconvergent. Clearly $B S(X) \leqslant A(X)$. On the other hand for each $\lambda>1 . A(X) / \lambda$ belongs to the defining set of $B S(X)$ and thus $B S(X) \geqslant A(X)$. Bynum [1] proved that $N(X) \leqslant$ $B S(X)$. To prove that $B S(X) \leqslant N(X)$, it suffices to show that for any bounded convex nonempty set $C$ with more than one point. there is a separable closed convex subset $C_{1}$ such that $R\left(C_{1}\right)=R(C)$. Indeed. if $\left\{x_{n}\right\}$ is a dense sequence in $C_{1}$ and $M$ is a number in the defining set of $B S(X)$, then

$$
M \leqslant A\left(\left\{x_{n}\right\}\right) / \lim _{n} \sup \left\|x_{n}-y\right\|=\frac{D\left(C_{1}\right)}{R\left(y C_{1}\right)} \leqslant \frac{D\left(C_{1}\right)}{R\left(C_{1}\right)} \leqslant \frac{D(C)}{R(C)} .
$$

To construct $C_{1}$, we start out with a sequence of points $\left\{z_{n}\right\}$ in $C$ such that $\lim _{n \rightarrow \infty} R\left(z_{n}, C\right)=R(C)$. Let $U_{1}=\operatorname{Co}\left(\left\{z_{n}\right\}\right)$. Let $V_{1}=\left\{x \in U_{1}: R\left(x, U_{1}\right)<R(C)\right\}$ and let $W_{1}$ be a countable dense subset of $V_{1}$. For each $x$ in $W_{1}$, let $D_{x}$ be a sequence of points in $C$ such that $R\left(x, D_{x}\right) \geqslant R(C)$. Let $X_{1}$ be the countable set $\cup\left\{D_{x}: x \in W_{1}\right\}$ and $U_{2}=\operatorname{Co}\left(U_{1} \cup X_{1}\right)$. We define similarly $V_{2}, W_{2}$ and $X_{2}$ from $U_{2}$ and continue this process to obtain an increasing sequence of convex sets $U_{1} \subset U_{2} \subset U_{3} \subset \cdots \subset U_{n} \subset \cdots$. Let $C_{1}=\overline{\operatorname{Co}}\left(\cup U_{n}\right) . C_{1}$ is separable. Since $R\left(z_{n}, C_{1}\right) \leqslant R\left(z_{n}, C\right)$ and $\lim _{n \rightarrow \infty} R\left(z_{n}, C\right)=R(C)$, we have $R\left(C_{1}\right) \leqslant R(C)$. From the way $U_{n}$ are constructed, $R\left(x, U_{n+1}\right) \geqslant R(C)$ for each $x \in U_{n}$. It follows that $R\left(C_{1}\right) \geqslant R(C)$ and the proof is complete.

For $0<\mu \leqslant \frac{1}{2}$ and $p>2$, denote by $x(\mu)$ the unique solution of the equation

$$
\lambda x^{p-1}-\mu-(\lambda x-\mu)^{p-1}=0
$$

in the interval $\mu / \lambda \leqslant x \leqslant 1$. Define $g(\mu), 0<\mu \leqslant 1$, by

$$
g(\mu)=\lambda \mu \frac{1+x(\lambda \wedge \mu)^{p-1}}{(1+x(\lambda \wedge \mu))^{p-1}}
$$

where $\lambda=1-\mu$. We proved in [4] the following inequality in $L^{p}(p>2)$ :

$$
\|\lambda x+\mu y\|^{p}+g(\mu)\|x-y\|^{p} \leqslant \lambda\|x\|^{p}+\mu\|x\|^{p}
$$

and that

$$
\sup _{0<\mu \leqslant 1} \frac{g(\mu)}{\mu}=\frac{1+\alpha^{p-1}}{(1+\alpha)^{p-1}}
$$

where $\alpha$ is the unique solution of

$$
(p-2) x^{p-1}+(p-1) x^{p-2}-1=0
$$

in the interval $0 \leqslant x \leqslant 1$. 
THEOREM 2. For $X=L^{p}, p>2$,

$$
N(X) \geqslant\left(1+\frac{1+\alpha^{p-1}}{(1+\alpha)^{p-1}}\right)^{1 / p} .
$$

Proof. For a closed convex bounded set $C$ in $X$, let $R$ and $D$ be the Chebyshev radius and the diameter of $C$ respectively. Let $z$ be the Chebyshev center of $C$. For $x$, $y$ in $C$ and $0<\mu \leqslant 1$, we have

$$
\|\lambda z+\mu y-x\|^{p}+g(\mu)\|z-y\|^{p} \leqslant \lambda\|z-x\|^{p}+\mu\|y-x\|^{p} .
$$

Taking sup over $x$ in $C$ and noting that $R \leqslant \sup \{\|\lambda z+\mu y-x\|: x \in C\}$, we obtain

$$
R^{p}+g(\mu)\|z-y\|^{p} \leqslant \lambda R^{p}+\mu \sup \left\{\|y-x\|^{p}: x \in C\right\} .
$$

It follows, after taking sup over $y$ in $C$, that $(\mu+g(\mu)) R^{p} \leqslant \mu D^{p}$ and hence

$$
\frac{D}{R} \geqslant\left(1+\sup _{0<\mu \leqslant 1} \frac{g(\mu)}{\mu}\right)^{1 / p}=\left(1+\frac{1+\alpha^{p-1}}{(1+\alpha)^{p-1}}\right)^{1 / p} .
$$

Therefore

$$
N(X) \geqslant\left(1+\frac{1+\alpha^{p-1}}{(1+\alpha)^{p-1}}\right)^{1 / p} .
$$

REMARK 1. For $p=3$ and 4, we have $\alpha=\sqrt{2}-1$ and $1 / 2$ and hence

$$
\left(1+\frac{1+\alpha^{p-1}}{(1+\alpha)^{p-1}}\right)^{1 / p}=(3-\sqrt{2})^{1 / 3} \text { and }(4 / 3)^{1 / 4}
$$

respectively.

\section{REFERENCES}

1. W. L. Bynum, Normal structure coefficients for Banach spaces, Pacific J. Math. 86 (1980), 427-436.

2. R. B. Holmes, $A$ course in optimization and best approximation, Lecture Notes in Math., vol. 257. Springer-Verlag, Berlin and New York, 1972.

3. T. C. Lim, Characterizations of normal structure, Proc. Amer. Math. Soc. 43 (1974), 313-319.

4. Theory, Method and Appl. (to appear).

Department of Mathematics, George Mason University, 4400 University Drive, Fairfax, VIRGINIA 22030 